\title{
A Study of Channel Coding and Decoding for Satellite Signals
}

\author{
Luo XianZhi \\ State Key Laboratory of Satellite Navigation System \\ and Equipment Technology \\ The $54^{\text {th }}$ Institute of China Electronic Technology \\ Corporation \\ Shijiazhuang, China \\ e-mail: 18632190780@163.com
}

\author{
Xie Jian \\ State Key Laboratory of Satellite Navigation System \\ and Equipment Technology \\ The $54^{\text {th }}$ Institute of China Electronic Technology \\ Corporation \\ Shijiazhuang, China \\ e-mail: xiejian8901@126.com
}

\begin{abstract}
Low-density parity check (LDPC) codes which have many encouraging characteristics, such as the lower complexities of the hardware implementation, closing to Shannon limit and no patent barrier, have gradually regained the interests of researchers. We present performance simulations in allusion to QC-LDPC codes. A fast double-recursion pipeline method which can significantly improve the encoding speed and a normalization min-sum algorithm are proposed in this paper. Simulations, hardware implementation and real test showed that QC-LDPC codes which has strong advantages on resource consumption, delay constraint and coding gain, could serves as an appealing alternative to new generation satellite navigation channel encoding and decoding.
\end{abstract}

Keywords-satellite navigation; channel coding; channel decoding; Low-Density Parity-Check code

\section{INTRODUCTION}

To enjoy navigation service, the receiver is required to acquire, track and complete data demodulation of navigation signals. However, the navigation signals are of low intensity and susceptible to interference when arrive at the earth after traveling $20,000 \mathrm{~km}$ overhead, which bring about difficulties for a receiver to acquire and track the navigation signal in addition to increased data demodulation BER. It is an important consideration to select channel coding solution of low complexity and good error-correcting capability in the system design of satellite navigation signals. With such strengths as low complexity of hardware implementation, performance close to Shannon limit, no patent barrier and very good errorcorrecting capability, LDPC codes have been used in many fields $s^{[1,2]}$. Moreover, along with the further research on LDPC codes, more advantages are discovered. To be specific, LDPC codes have the following strengths:

(1) As linear block code, LDPC codes have good flexibility and are applicable to a great variety of channels.

(2) In the decoding process, the iteration decoding design and parallel decoding design are combined together for enhanced decoding capability.

(3) Nonadjacent variable nodes are involved in the decoding decision with the same check equation when decoding, which provides burst error resistance to some extent and avoids introduction of interleaver as well as the delay brought along with interleaver.
(4) The computation complexity will not grow sharply along with the increase of code length because of the sparsity of check matrix, which is favorable for the coding and decoding implementation of long codes.

(5) Lower complexity of hardware implementation, especially in favor of the quasi-cyclic check matrix ${ }^{[3,6]}$.

\section{SIMULATION OF QC-LDPC PERFORMANCE}

To implement high-gain coding at $1 / 2$ code rate, a coding scheme of variable frame length was devised considering the design uncertainty of frame format for navigation signal. The encoding scheme consists of basic check matrix $H_{b}$ and $\mathrm{Z}$ factor $Z_{f}$, with the key parameters as follows:

TABLE I. 1/2 LDPC CODING SCHEME

\begin{tabular}{|c|c|}
\hline Items & Parameters \\
\hline Code rate $\mathrm{R}$ & $1 / 2$ \\
\hline Coding scheme & QC - LDPC \\
\hline Z factor & $24 \sim 96$, in steps of 4 \\
\hline Message length $\mathrm{k}$ & $\mathrm{Z}$ factor $\times 12$ \\
\hline
\end{tabular}

TABLE II. 1/2 LDPC PARITY-CHECK MATRIX $H_{b}$

$\begin{array}{llllllllllllllllllllllll}-1 & 94 & 73 & -1 & -1 & -1 & -1 & -1 & 55 & 83 & -1 & -1 & 7 & 0 & -1 & -1 & -1 & -1 & -1 & -1 & -1 & -1 & -1 & -1\end{array}$ $\begin{array}{llllllllllllllllllllllll}-1 & 27 & -1 & -1 & -1 & 22 & 79 & 9 & -1 & -1 & -1 & 12 & -1 & 0 & 0 & -1 & -1 & -1 & -1 & -1 & -1 & -1 & -1 & -1\end{array}$ $\begin{array}{llllllllllllllllllllllll}-1 & -1 & -1 & 24 & 22 & 81 & -1 & 33 & -1 & -1 & -1 & 0 & -1 & -1 & 0 & 0 & -1 & -1 & -1 & -1 & -1 & -1 & -1 & -1\end{array}$ $\begin{array}{llllllllllllllllllllllll}61 & -1 & 47 & -1 & -1 & -1 & -1 & -1 & 65 & 25 & -1 & -1 & -1 & -1 & -1 & 0 & 0 & -1 & -1 & -1 & -1 & -1 & -1 & -1\end{array}$ $\begin{array}{lllllllllllllllllllllllll}-1 & -1 & 39 & -1 & -1 & -1 & 84 & -1 & -1 & 41 & 72 & -1 & -1 & -1 & -1 & -1 & 0 & 0 & -1 & -1 & -1 & -1 & -1 & -1\end{array}$ $\begin{array}{llllllllllllllllllllllll}-1 & -1 & -1 & -1 & 46 & 40 & -1 & 82 & -1 & -1 & -1 & 79 & 0 & -1 & -1 & -1 & -1 & 0 & 0 & -1 & -1 & -1 & -1 & -1\end{array}$ $\begin{array}{lllllllllllllllllllllllllll}-1 & -1 & 95 & 53 & -1 & -1 & -1 & -1 & -1 & 14 & 18 & -1 & -1 & -1 & -1 & -1 & -1 & -1 & 0 & 0 & -1 & -1 & -1 & -1\end{array}$ $\begin{array}{llllllllllllllllllllllll}-1 & 11 & 73 & -1 & -1 & -1 & 2 & -1 & -1 & 47 & -1 & -1 & -1 & -1 & -1 & -1 & -1 & -1 & -1 & 0 & 0 & -1 & -1 & -1\end{array}$ $\begin{array}{llllllllllllllllllllllll}12 & -1 & -1 & -1 & 83 & 24 & -1 & 43 & -1 & -1 & -1 & 51 & -1 & -1 & -1 & -1 & -1 & -1 & -1 & -1 & 0 & 0 & -1 & -1\end{array}$ $\begin{array}{llllllllllllllllllllllll}-1 & -1 & -1 & -1 & -1 & 94 & -1 & 59 & -1 & -1 & 70 & 72 & -1 & -1 & -1 & -1 & -1 & -1 & -1 & -1 & -1 & 0 & 0 & -1\end{array}$ $\begin{array}{llllllllllllllllllllllll}-1 & -1 & 7 & 65 & -1 & -1 & -1 & -1 & 39 & 49 & -1 & -1 & -1 & -1 & -1 & -1 & -1 & -1 & -1 & -1 & -1 & -1 & 0 & 0\end{array}$ $\begin{array}{llllllllllllllllllllllll}43 & -1 & -1 & -1 & -1 & 66 & -1 & 41 & -1 & -1 & -1 & 26 & 7 & -1 & -1 & -1 & -1 & -1 & -1 & -1 & -1 & -1 & -1 & 0\end{array}$

The encoding scheme of two frame lengths are devised on the basis of $1 / 2$ LDPC encoding scheme, $(480,960)$ and $(960,1920)$.

Considering the possible selection of message length, the scheme for different message lengths was verified by 
means of simulation. The result is as shown in Fig .1.

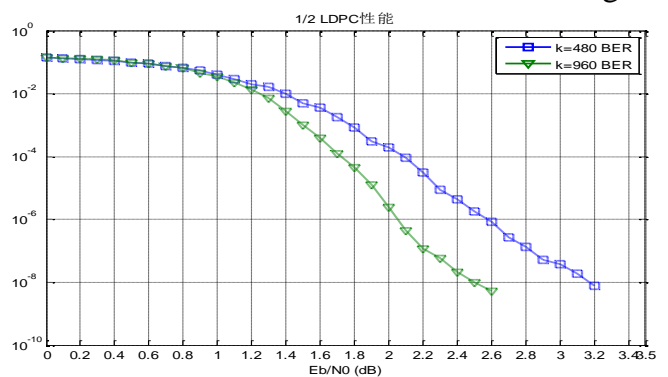

Figure 1. BER performance of LDPC coding for different frame lengths

Based on an analysis and comparison of the simulation results, it was found that $1 / 2 \mathrm{LDPC}$ scheme required the SNR to be $3.2 \mathrm{~dB}$ when the message length was 480 bits and the BER was $1 \mathrm{E}-8$, which achieved $8.8 \mathrm{~dB}$ coding gain in comparison with the non-coding scheme. It required the SNR to be $2.6 \mathrm{~dB}$ when the message length was 960 bits and the BER was $1 \mathrm{E}-8$, which achieved $9.4 \mathrm{~dB}$ coding gain in comparison with the non-coding scheme. In other words, the 960 bits message length improve the coding gain by $0.6 \mathrm{~dB}$ comparing with the 480 bits message length.

\section{LDPC ENCODING ALGORITHM AND IMPLEMENTATION}

\section{A. Two-way Recursion Fast Coding Algorithm Selecting a Template $^{[7,8]}$}

The QC-LDPC codes described above adopt dualdiagonal parity structure, which makes it possible to complete fast coding in a way of cyclic recursion. A code $\mathbf{c}$ is divided into input information sequence $\mathbf{s}$ and check sequence $\mathbf{p}$ that is generated in coding process. $\mathbf{s}$ and $\mathbf{p}$ are further decomposed into $k_{b}$ and $m_{b}$ groups of $z$ dimension sub-vectors. Therefore, a code may be expressed as $\mathbf{c}=[\mathbf{s} \mid \mathbf{p}]=\left[\mathbf{s}_{0}, \mathbf{s}_{1}, \mathrm{~L}, \mathbf{s}_{\mathbf{k}_{\mathbf{b}}-1}, \mathbf{p}_{0}, \mathbf{p}_{1}, \mathrm{~L}, \mathbf{p}_{\mathbf{m}_{\mathbf{b}}-1}\right]$, where $\mathbf{L}_{\mathbf{i}, \mathbf{j}}$ represents the sub-matrix corresponding to the element defined by Row $i$ and Column $j$ in matrix $\mathbf{H}_{b_{1}}$. Let's assume $h_{b}(0)=h_{b}\left(m_{b-1}\right)=l, h_{b}(x)=l_{x}$ and

$$
\mathbf{b}_{\mathbf{i}}=\sum_{j=0}^{k_{b}-1} \mathbf{L}_{\mathbf{i}, j} \mathbf{s}_{\mathbf{j}} \quad\left(0 \leq i \leq m_{b}-1\right)
$$

Then, due to $\mathbf{H} \times \mathbf{c}^{\mathbf{T}}=\mathbf{0}$, it is possible to deduce that

$$
\begin{aligned}
& \mathbf{b}_{0}+\mathbf{p}_{0}{ }^{(l)}+\mathbf{p}_{1}=\mathbf{0} \\
& \mathbf{b}_{\mathbf{i}}+\mathbf{p}_{\mathbf{i}}+\mathbf{p}_{\mathbf{i}+1}=\mathbf{0} \quad\left(1 \leq i \leq m_{b}-2, i \neq x\right) \\
& \mathbf{b}_{\mathbf{x}}+\mathbf{p}_{0}{ }^{\left(l_{x}\right)}+\mathbf{p}_{\mathbf{x}}+\mathbf{p}_{\mathbf{x}+1}=\mathbf{0} \\
& \mathbf{b}_{\mathbf{m}_{\mathbf{b}}-1}+\mathbf{p}_{0}{ }^{(l)}+\mathbf{p}_{\mathbf{m}_{\mathbf{b}}-1}=\mathbf{0}
\end{aligned}
$$

Where, $\mathbf{p}_{i}^{(l)}$ represents the vector of $\mathbf{p}_{i}$ after shifting $l$ times rightwards circularly.

If the $m_{b}$ equations (2) through (5) corresponding to different rows are added together, we will obtain:

$$
\mathbf{p}_{0}{ }^{\left(l_{x}\right)}=\sum_{i=0}^{m_{b}-1} \mathbf{b}_{\mathbf{i}}
$$

After $\mathbf{p}_{0}$ is worked out, it is possible to figure out the following equation by means of forward recursion on the basis of Equation (2)

$$
\mathbf{p}_{1}=\mathbf{b}_{0}+\mathbf{p}_{0}{ }^{(l)}
$$

In the meantime, it is possible to figure out the following equation by means of backward recursion on the basis of Equation (5).

$$
\mathbf{p}_{\mathbf{m}_{\mathbf{b}}-1}=\mathbf{b}_{\mathbf{m}_{\mathbf{b}}-1}+\mathbf{p}_{0}^{(l)}
$$

After $\mathbf{p}_{1}, \mathbf{p}_{\mathbf{m}_{\mathbf{b}}-1}$ is figured out, go on with the forward recursion to work out:

$$
\mathbf{p}_{2}=\mathbf{b}_{1}+\mathbf{p}_{1}
$$

In the meantime, proceed with backward recursion to work out:

$$
\mathbf{p}_{\mathbf{m}_{\mathbf{b}}-2}=\mathbf{b}_{\mathbf{m}_{\mathbf{b}}-2}+\mathbf{p}_{\mathbf{m}_{\mathbf{b}}-1}
$$

Accordingly, it is possible to work out the intermediate check vector at last by means of two-way recursion.

$$
\mathbf{p}_{\mathbf{m}_{\mathrm{b}} / 2}=\mathbf{b}_{\mathbf{m}_{\mathbf{b}} / 2}+\mathbf{p}_{\left(\mathbf{m}_{\mathbf{b}} / 2\right)+1}
$$

The sequential order of check vectors that are worked out with this two-way recursive algorithm is $\left(\mathbf{p}_{0}\right),\left(\mathbf{p}_{1}, \mathbf{p}_{\mathbf{m}_{\mathbf{b}}-1}\right), \mathrm{L},\left(\mathbf{p}_{\left(\mathbf{m}_{\mathrm{b}} / 2\right)-1}, \mathbf{p}_{\left(\mathbf{m}_{\mathbf{b}} / 2\right)+1}\right)$ and $\left(\mathbf{p}_{\mathbf{m}_{\mathbf{b}} / 2}\right)$, where the parentheses mean the two check vectors may be solved simultaneously. Finally, it is possible to acquire the code in the form required by the system by combining the check vectors and the information vectors that are worked out together. In terms of hardware implementation, this two-way recursive coding algorithm is characterized by low computation complexity, high degree of parallelism, less resource consumption and low cabling complexity.

\section{B. Pipelining Implementation of Quick Two-way Recursion Pipeline Algorithm ${ }^{[9,10]}$}

After all the $\mathbf{b}_{\mathbf{i}}$ vectors are figured out, work out the check vector $\mathbf{p}_{\mathbf{0}}$ using Equation (6). And then, work out all the other check vectors $\left(\mathbf{p}_{1}, \mathbf{p}_{\mathbf{m}_{\mathbf{b}}-1}\right), \mathbf{L},\left(\mathbf{p}_{\left(\mathbf{m}_{\mathbf{b}} / 2\right)-1}, \mathbf{p}_{\left(\mathbf{m}_{\mathbf{b}} / 2\right)+1}\right)$ and $\left(\mathbf{p}_{\mathbf{m}_{\mathbf{b}} / 2}\right)$ one by one with the two-way recursive algorithm described in Section 1. Based on an analysis of the computational process of two-way recursion encoding algorithm, we developed a quick pipeline algorithm for the computation of $\mathbf{p}_{1}, \mathrm{~L}, \mathbf{p}_{\mathbf{m}_{\mathrm{b}}-1}$. It can be seen from equations (7) through (11) that $\mathbf{b}_{\mathbf{i}}$ variables computation of different $\mathbf{p}_{\mathbf{i}}$ vectors with two-way recursive algorithm. $\mathbf{b}_{0}, \mathbf{b}_{\mathbf{m}_{\mathbf{b}}-1}$ is required for computation of $\mathbf{p}_{1}, \mathbf{p}_{\mathbf{m}_{\mathbf{b}}-1} ; \mathbf{p}_{2}, \mathbf{p}_{\mathbf{m}_{\mathbf{b}}-2}$ are required for computation of $\mathbf{b}_{1}, \mathbf{b}_{\mathbf{m}_{\mathbf{b}}-2}$, and so on Therefore, there is no access conflict for $\mathbf{b}_{\mathbf{i}}$ data in the parallel computation of $\mathbf{p}_{\mathbf{i}}$. However, in the computation process of $\mathbf{p}_{\mathbf{i}}$, the nesting dependence on its own makes it impossible for $\mathbf{p}_{\mathbf{i}}$ to complete synchronous computation. 
Besides, the computation of $\mathbf{p}_{1}, \mathbf{p}_{\mathbf{m}_{\mathbf{b}}-1}$ depends on $\mathbf{p}_{0}$ and the computation of $\mathbf{p}_{2}, \mathbf{p}_{\mathbf{m}_{\mathbf{b}}-2}$ depends on $\mathbf{p}_{1}, \mathbf{p}_{\mathbf{m}_{\mathbf{b}}-1}$. Such nesting dependence forces the computation of $\mathbf{p}_{\mathbf{i}}$ to start from the both ends of the dual diagonals towards the middle in series. However, upon a further analysis, it is found that this dependency relationship is virtually nesting dependence between components of different $\mathbf{p}_{\mathbf{i}}$ vectors rather than between $\mathbf{p}_{\mathbf{i}}$ vectors. After the first components of $\mathbf{p}_{1}, \mathbf{p}_{\mathbf{m}_{\mathbf{b}}-1}$ are figured out, it is possible to compute the first components of $\mathbf{p}_{2}, \mathbf{p}_{\mathbf{m}_{\mathbf{b}}-2}$ and not necessary to wait the completion of the entire $\mathbf{p}_{1}, \mathbf{p}_{\mathbf{m}_{\mathbf{b}}-1}$ vector computation. Therefore, it is feasible to carry out approximative synchronous computation of $\mathbf{p}_{\mathbf{i}}$ by inserting the pipeline algorithm. The pipeline work flow for computation of check vector is as shown in Fig .2 when $\mathbf{m}_{\mathbf{b}}=8$. Fig .3 shows the clock sequence in which the check vectors were worked out. It needs $\mathrm{z}+m_{b}-1$ clock cycles altogether to work out $\mathbf{p}_{1}, \mathrm{~L}, \mathbf{p}_{\mathbf{m}_{\mathbf{b}}-1}$ with this quick pipeline algorithm, which requires only $m_{b}-1$ more clock cycles than $\mathrm{z}$ that is needed for purely synchronous computation of $\mathbf{p}_{\mathbf{i}}$. Just for comparison, it needs a total of $\mathrm{z} \times m_{b}$ clock cycles if the pipeline algorithm is not used. Another benefit brought along with this pipeline architecture is the synchronous output of $\mathbf{p}_{1}$, i.e., instant output at the end of $\mathbf{p}_{1}$ check-bit computation. It does not require buffer memory for vector $\mathbf{p}_{1}$, consequently to save storage resource. The encoder presented here in this paper adopts such an output design.

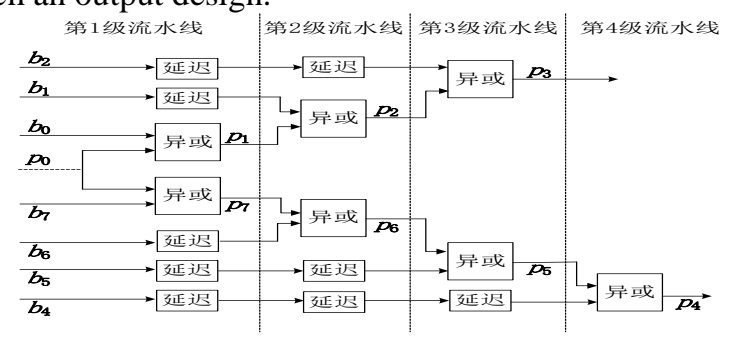

Figure 2. Pipeline workflow in computation of check vectors when $m_{b}$ $=8$

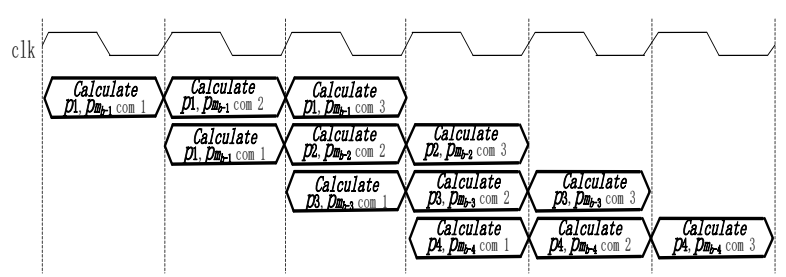

Figure 3. Computation timing diagram of parity-check vectors

\section{PRePARE Your PAPER Before StYling}

\section{A. BP Algorithm of Logarithm Domain(LLR-BP)}

Decoding algorithm is purposed to acquire correct codes from the received messages as well as the relationship between nodes and parity-check nodes. For the convenience of description, the following notations are defined in the first place.
$V_{j}=$ Variable node linked to check node $f_{j}$

$V_{j} / i=$ Variable node linked to check node $f_{j} \backslash$ Variable node $c_{i}$

$=$ Check node linked to variable node $c_{i}$

$=$ Check node linked to variable node $c_{i} \backslash$ Check node $f_{j}$

$M v(\sim i)=$ Messages coming from all the variable nodes except for $c_{i}$

=Messages coming from all the check nodes except for $f_{j}$

$p_{i}=\operatorname{Pr}\left(c_{i}=1 \mid y_{i}\right)$

$S_{i}=$ All the parity-check equations containing $c_{i}$ satisfy this event.

$q_{i j}=\operatorname{Pr}\left(c_{i}=b \mid S_{i}, y_{i}, M c(\sim j)\right), \quad b \in\{0,1\}$

$r_{i j}(b)=\operatorname{Pr}\left(\quad\right.$ Parity-check equation $f_{i}$ satisfies $\left.c_{i}=b, M v(\sim i)\right), \quad b \in\{0,1\}$ 。

For additive white Gaussian noise channel, let's set $x_{i}=1-2 c_{i}$ to serve as the binary value of the $i$ th transmission. Thus, $x_{i}=+1(-1)$ when $c_{i}=0(1)$. After that, replace $c_{i}$ with $x_{i}$. Now let's define the log likelihood ratio.

$$
\begin{aligned}
& L\left(c_{i}\right)=\log \left(\frac{\operatorname{Pr}\left(c_{i}=0 \mid y_{i}\right)}{\operatorname{Pr}\left(c_{i}=1 \mid y_{i}\right)}\right) \\
& L\left(r_{j i}\right)=\log \left(\frac{r_{j i}(0)}{r_{j i}(1)}\right) \\
& L\left(q_{i j}\right)=\log \left(\frac{q_{j i}(0)}{q_{j i}(1)}\right) \\
& L\left(Q_{i}\right)=\log \left(\frac{Q_{i}(0)}{Q_{i}(1)}\right)
\end{aligned}
$$

Thereby, it is possible to know the main steps of the logarithm-domain BP decoding algorithm(LLR-BP) as follows:

\section{1) Initialization}

For every received variable node, work out the initial channel information with the following equation.

$$
L\left(q_{i j}\right)=L\left(c_{i}\right)=2 y_{i} / \sigma^{2}
$$

In the meantime, initialize the iteration times.

2) Updating check node

For all the check nodes $f_{j}$ and their adjacent variable nodes $c_{i} \in V_{j}$, work out the messages $\left\{L\left(r_{i j}\right)\right\}$ transmitted to the check nodes from the variable nodes.

$$
\begin{aligned}
L\left(r_{j i}\right) & =\prod_{i^{\prime} \in V_{j} \backslash i} \alpha_{i^{\prime} j} \cdot 2 \tanh ^{-1}\left[\prod_{i^{\prime} \in V_{j} \backslash i} \tanh \left(\frac{1}{2} \beta_{i^{\prime} j}\right)\right] \\
& =\prod_{i^{\prime} \in V_{j} \backslash i} \alpha_{i^{\prime} j} \cdot 2 \tanh ^{-1} \log ^{-1} \log \left[\prod_{i^{\prime} \in V_{j} \backslash i} \tanh \left(\frac{1}{2} \beta_{i^{\prime} j}\right)\right] \\
& =\prod_{i^{\prime} \in V_{j} \backslash i} \alpha_{i^{\prime} j} \cdot 2 \tanh ^{-1} \log ^{-1} \sum_{i^{\prime} \in V_{j} \backslash i} \log \left[\tanh \left(\frac{1}{2} \beta_{i^{\prime} j}\right)\right]
\end{aligned}
$$

Where, $\alpha_{i^{\prime} j}=\operatorname{sign}\left(q_{i^{\prime} j}\right)$ and $\beta_{i^{\prime} j}=\left|q_{i^{\prime} j}\right|$.

3) Updating variable nodes 


$$
L\left(q_{i j}\right)=L\left(c_{i}\right)+\sum_{j^{\prime} \in C_{i} \backslash j} L\left(r_{j^{\prime} i}\right)
$$

4) LQ updating

On completion of all the non-zero node computation, work out $L Q$ for all the row number $i$.

$$
L\left(Q_{i j}\right)=L\left(c_{i}\right)+\sum_{j \in C_{i}} L\left(r_{j i}\right)
$$

5) Decision processing. Work out $\hat{c}_{i}$ for each row:

$$
\hat{c}_{i}=\left\{\begin{array}{cc}
1 & L\left(Q_{i}\right)<0 \\
0 & \text { other }
\end{array}\right.
$$

Stop iterating if $\hat{c} H^{T}=0$ or the iteration reaches the maximum number; otherwise, go back to Step 2 to start next iteration.

\section{B. Modification of LLR-BP Algorithm}

Since tanh and its inverse operation are involved in the updating equation of check node in the LLR-BP algorithm, hardware implementation is not easy to achieve but with look-up table method.

MinSum algorithm is a decoding algorithm with regard to the excessive complexity of $\varphi(x)$ that is used in the check node updating in LLR-BP decoding algorithm. Considering the information updating characteristic of check nodes and variable nodes in the probability-domain and logarithm-domain BP decoding algorithm, MinSum algorithm replaces the log computation, multiplication and division with addition, subtraction and comparison. These changes lower the decoding complexity significantly while the decoding performance does not run down much in comparison with the LLR-BP decoding algorithm.

let's define $\varphi(x)=-\log [\tanh (x / 2)]=\log \left(e^{x}+e^{-x}-1\right)$. According to the function character, it is known that $\varphi^{-1}(x)=\varphi(x)$ and $x>0$. And then,

$$
L\left(r_{j i}\right)=\prod_{i^{\prime} \in V_{j} \backslash i} \alpha_{i^{\prime} j} \varphi\left[\sum_{i^{\prime} \in V_{j} \backslash i} \varphi\left(\beta_{i^{\prime} j}\right)\right]
$$

Because it is hard to achieve hardware implementation for function $\varphi(x)$, it is required to initialize the function by means of approximation for practical application. Function $\varphi(x)$ has one characteristic as follows: the function value falls pretty fast along with the growth of $x$. Therefore, function $\varphi\left[\sum_{i^{\prime} \in V_{j} \backslash i} \varphi\left(\beta_{i^{\prime} j}\right)\right]$ almost depends on the minimum of $\beta_{i^{\prime} j}$ completely, that is, $\sum_{i^{\prime} \in V_{j} \backslash i} \varphi\left(\beta_{i^{\prime} j}\right) \approx \varphi\left(\min _{i^{\prime} \in V_{j} \backslash i} \beta_{i^{\prime} j}\right)$. For this reason, the updating of check node may be simplified as:

$$
L\left(r_{j i}\right)=\left(\prod_{i^{\prime} \in V_{j} \backslash i} \operatorname{sign}\left(L q_{i j}\right)\right) \min _{i^{\prime} \in V_{j} \backslash i}\left(\beta_{i^{\prime} j}\right)
$$

After processing by this step, the hardware implementation will be facilitated, for the computational workload and the memory capacity is saved a lot. This processing step is also the main difference between MinSum algorithm and LLR-BP algorithm. However, this characteristic also brings about performance loss to some extent. The performance loss is especially considerable at low code rate. To enhance the performance of MinSum algorithm, several modified MinSum algorithms are proposed as follows.

Modified Algorithm 1: Normalized-MS(NormalizedMinSum algorithm)
At the updating step of check nodes, the updating equation becomes:

$$
\begin{aligned}
& \Lambda_{m n}^{(k)}=\alpha \cdot \prod_{n^{\prime} \in N(m) \backslash n} \operatorname{sign}\left(\lambda_{m n}^{(k-1)}\right) \cdot \min _{n^{\prime} \in N(m) \backslash n}\left|\lambda_{m n}^{(k-1)}\right| \\
& L\left(r_{j i}\right)=\alpha \cdot \prod_{i^{\prime} \in V_{j} \backslash i} \operatorname{sign}\left(L q_{i j}\right) \min _{i^{\prime} \in V_{j} \backslash i}\left(\beta_{i^{\prime} j}\right)
\end{aligned}
$$

Where, $\alpha$ is usually a constant less than 1 , of which the specific value must be analyzed with density evolution analytic technique based on different SNRs and iteration times. This modified algorithm is purposed to minify the updating value of check nodes with $\alpha$ so as to approach the updating value of check nodes in LLR-BP algorithm.

Modified Algorithm 2: Offset-MS(Offset-based MinSum Algorithm)

At the updating step of check nodes, the updating equation becomes:

$$
\begin{aligned}
& \Lambda_{m n}^{(k)}=\prod_{n^{\prime} \in N(m) \backslash n} \operatorname{sign}\left(\lambda_{m n^{\prime}}^{(k-1)}\right) \cdot \max \left\{\min _{n^{\prime} \in N(m) \backslash n}\left|\lambda_{m n}^{(k-1)}\right|-\beta, 0\right\} \\
& L\left(r_{j i}\right)=\prod_{i^{\prime} \in V_{j} \backslash i} \operatorname{sign}\left(L q_{i j}\right) \cdot \max \left\{\min _{i^{\prime} \in V_{j} \backslash i}\left(\beta_{i^{\prime} j}\right)-\eta, 0\right\}
\end{aligned}
$$

Where, $\eta$ is usually a positive constant, which must be optimized with density evolution analytic technique based on different SNRs and iteration times. This modified algorithm is purposed to determine if the updating value of check nodes in MinSum algorithm is useful or not by means of comparing both $\min _{n \in N(m) \backslash n}\left|\lambda_{m n}^{(k-1)}\right|$ and $\eta$, so as to approach the updating value of check nodes in LLR-BP algorithm.

In this modified algorithm, the check nodes will not be updated when $\min _{n \in N(m) \backslash n}\left|\lambda_{m n^{(k-1)}}\right| \leq \eta$; therefore, its algorithm performance is somewhat worse than NMS algorithm performance. However, this algorithm is easy to be implemented, for it does not require multiply operation.

\section{PERORMANCE EVAluationOF CODINGAND DECODING MODLUE}

The resources occupied by the coding module are as shown below:

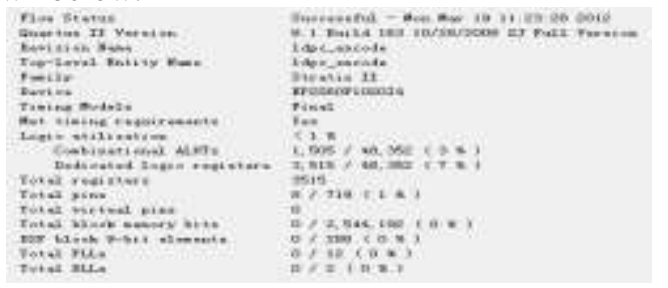

Figure 4. The resources occupied by the coding module

Adaptive look-up table(ALUT) and memory are two important indicators in FPGA resource evaluation. The above figure showed that a total of 1505 ALUTs were occupied when EP2S60F1020I4 chip was used for coding implementation, which accounted for $3 \%$ of the total chip resources.

The resources occupied by the decoding module are as shown below when Normalized-MS algorithm is adopted. 


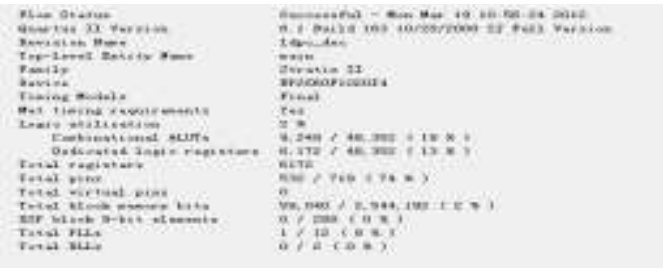

Figure 5. The resources occupied by the decoding module

ALUT and memory are two important indicators in FPGA resource evaluation. The above figure showed that a total of 9249 ALUTs were occupied when EP2S60F1020I4 chip was used for decoding implementation, which accounted for $19 \%$ of the total chip resources; a total of 59040 bit memory was occupied, which accounted for $2 \%$ of the total chip resources.

\section{PERORMANCE EVALUATIONOF CODINGAND DECODING MODLUE}

In terms of coding delay, fast coding technique is used so that the coding process can be completed in about 20 clock cycles. The coding delay is approximately $2 \mathrm{~ms}$ when the work clock of the coding module is $10 \mathrm{KHz}$.

As for decoding delay, the decoding process needs about 544,450 clock cycles under the condition of 50 iterations and using the Normalized-MS algorithm. When the work clock of the decoding module is $40 \mathrm{MHz}$, the decoding delay is $13.6 \mathrm{~ms}$; when the work clock is $60 \mathrm{MHz}$, the decoding delay is $9.1 \mathrm{~ms}$.

\section{Evaluation OF DCECODING PERFORMANCE}

A separate test was given to the LDPC decoding module and the test results were compared with MATLAB simulation curve to verify the module correctness and estimate the implementation cost of coding and decoding. The test block diagram is as shown in Fig .6.

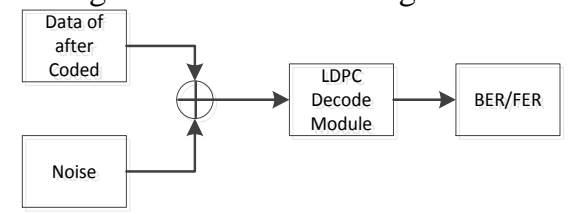

Figure 6. Block diagram of LDPCdecoding performance test

The demonstration tests were given when the $\mathrm{Eb} / \mathrm{N} 0$ was $0,0.5,1,1.5$ and 2 respectively. In the testing process, MATLAB was used for generation after the Eb/N0 was determined. A fixed frame data were inserted with noise after coding. On completion of 8bit quantization, the signal vector and the noise vector were stored in a ROM register. And then, read the data from two ROMs at $10 \mathrm{KHz}$ clock. Added them together before sending them into the LDPC decoding module. Acquired the BER and FER at the end of decoding. Compared the test results with the simulation results generated by MATLAB to end up the validation test of LDPC decoding module. The test results are as shown in Fig .7.

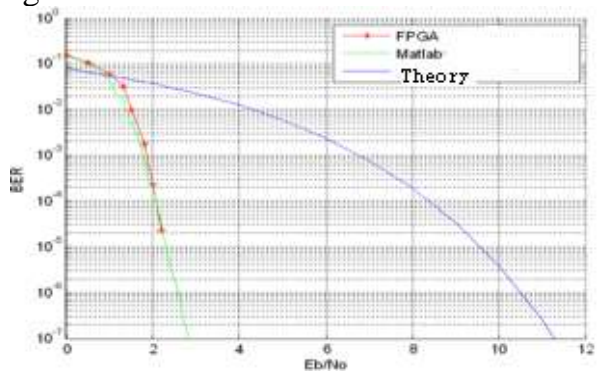

Figure 7. Performance test of LDPC decoding module

\section{CONCULUSION}

Conclusively, LDPC coding and decoding module is capable of satisfying the design requirements of navigation signal in terms of resource expenditure, delay constraint and coding gain.

\section{REFERENCES}

[1] R.G.WEI,H.CHEN, J.HQIU, Z.S.HAO, G.X.LEI,Research onLDPC Codes Decoding Algorithm for High Data Rate Transmission,Radio Engineering, vol.41, No.3,Mar.2011,pp2022.

[2] D.J.C. MacKay and R.M. Neal,"Near Shannon Limit Perform-ance of Low Density Parity Check Codes,"Electron.Lett,vol.32,Aug.1996,pp1645-1646, DOI:10.1049/el:19961141

[3] L.Z. W, LCHEN, L.Q.ZENG, et al, "Efficient Encoding of Quasi-Cyclic Low-Density Parity-Check Codes,"IEEE Transactions on Communications, vol.54, No.1, 2006,pp71-81.

[4] R.G.GALLAGE, "Low Density Parity Check Codes,'IRETransactions on Information Theory, vol.8, No.8, 1962,pp208-220

[5] Y.KOU, S.LIN, M.P.C . FOSSORIER,"Low-density Parity check Codes Based on Finite Geometries:a Rediscovery and New Results,"IEEE Transactions on Information Theory, vol.47, No.7, 2001,pp2711-2736.

[6] T.J. Richardson, "Efficient Encoding of Low-density Parity-check Codes,"IEEE Trans on Information Theory, vol.47, No.2, 2001,pp638-656,doi:10.1109/18.910579.

[7] R.J. SUN, "Research and application of LDPC decoding method," dissertation of Nanjing University of Science and Technology,2012.

[8] J.P. YANG, Q.C CHEN, "Design and Implementation of 802.16e Standard LDPC Decoder,"COMMUNICATIONS TECHNOLOGY, vol.43, No.5, 2010, pp18-19.

[9] S.B. HE, C.H. LIU, Y.M. LIN, "Application of LDPC Codes in Satellite Navigation Systems", SPACECRAFT ENGINEERING, vol.18, No.3, 2009.05, pp72-76

[10] H. QIAO, W. GUAN, M.K. DONG, H.G. XIANG, "Design and Implementation of LDPC Decoder with High Throughput", Acta Scientiarum Naturalium Universitatis Pekinensis, vol.2, No.2, 2007, pp1-6. 